\title{
Sustained effects of intralymphatic pollen-specific immunotherapy on Japanese cedar pollinosis*
}

\author{
Tetsuya Terada', Shyuji Omura', Yusuke Kikuoka', Manabu Suzuki', Yuko \\ Inaka', Takaki Inui', Masaya Matsuda², Takeshi Nabe², Ryo Kawata' \\ 1 Department of Otolaryngology, Head and Neck Surgery, Osaka Medical College, Osaka, Japan \\ 2 Laboratory of Immunopharmacology, Faculty of Pharmaceutical Sciences, Setsunan University, Osaka, Japan
}

Rhinology 58: 3, 241 - 247, 2020

https://doi.org/10.4193/Rhin19.301

*Received for publication:

August 30, 2019

Accepted: December 3, 2019

\begin{abstract}
Background: Intralymphatic immunotherapy (ILIT) for allergic patients requires only a few intralymphatic injections of the allergen. However, the effectiveness and safety for Japanese cedar pollinosis are unclear. The objectives of this study were to clarify whether and how long ILIT is effective for pollinosis, and its safety.
\end{abstract}

Methods: In an open pilot investigation followed by a double-blind, placebo-controlled study, patients with Japanese cedar pollinosis received 3 intralymphatic inguinal injections of the pollen extracts before the first pollen season. The symptom medication score (SMS), nasal provocation testing and scoring visual analogue scale (VAS) were assessed after the first-third seasons.

Results: (1) Although mild adverse events were induced at the injected site, severe adverse events were not noted. (2) During the latter part of the first season, ILIT-treated patients $(n=12)$ tended to show improved SMS compared to placebo-treated $(n=6)$ without statistical significance. When assessed by nasal provocation testing and VAS scoring after the first season, the effectiveness of ILIT was significant. (3) The effects of ILIT continued until the second or third season. (4) Neither allergen-specific antibodies nor Treg/Breg cells changed in the peripheral blood.

Conclusions: ILIT was safe and effective for Japanese cedar pollinosis. The clinical effects remained for 1-2 years.

Key words: allergy, IgE, intralymphatic immunotherapy, pollinosis, Treg

\section{Introduction}

The prevalence of Japanese cedar pollinosis has steadily increased in Japan, reaching more than $30 \%$ of the Japanese population ${ }^{(1)}$. Although pollinosis is not life-threatening, it markedly reduces the QOL of patients and affects productivity, leading to economic and social problems ${ }^{(2-6)}$. The treatment of pollinosis includes pharmacotherapy, surgery, and immunological therapies. Allergen-specific immunotherapy is the only treatment that reduces symptoms, improves QOL, prevents new sensitization, and reduces the development of asthma in allergic rhinitis patients. The most common immunotherapy is subcutaneous immunotherapy (SCIT), which may require more than 50 injections of allergen extracts over 3 to 5 years ${ }^{(3)}$. Sublingual immunotherapy (SLIT) has also been demonstrated to be effective for pollinosis ${ }^{(7-9)}$. Although SLIT does not require injections, patients have to sublingually take allergen extracts every day for several years, and the treatment duration cannot be shortened. Because of this disadvantage, only $5 \%$ of allergic patients undergo antigen-specific immunotherapy ${ }^{(10,11)}$. Thus, allergen immunotherapy through other administration routes should be investigated.

Intralymphatic immunotherapy (ILIT) for some allergens, such as cat dander and pollen, has also been demonstrated to be effective for allergic rhinitis ${ }^{(12-16)}$. ILIT induces tolerance to a specific allergen more rapidly than SCIT and SLIT after only 3 injections of allergen extracts, and does not induce serious adverse effects. Antigens administered into the lymph node may be efficiently delivered to antigen-presenting cells, followed by the activation of T cells and B cells. Thus, ILIT may be advantageous because only a few injections of a small amount of allergen extract are 
Table 1. Subjects' characteristics.

\begin{tabular}{lcc} 
& Placebo & ILIT \\
$\mathrm{N}$ & 6 & 12 \\
Sex (Male/Female) & $2 / 4$ & $4 / 8$ \\
\hline Age (years) & $45.5(39-54)$ & $40.5(30-65)$ \\
\hline Total IgE (IU/ml) & $158.7(86.2-249)$ & $150.6(13.5-482)$ \\
\hline Antigen specific lgE (IUA/ml) & $20.9(6.39-53.8)$ & $13.2(2.69-41.4)$
\end{tabular}

necessary to achieve clinical effectiveness. However, compared with studies on SCIT and SLIT, there are few demonstrating the clinical efficacy and safety of ILIT, including its efficacy and safety for Japanese cedar pollinosis. In addition, it is unknown how effective ILIT is for subsequent pollen seasons.

The mechanisms underlying the clinical effectiveness of allergen immunotherapy are unclear. Some mechanisms, including the decrease in antigen-specific lgE and increase in antigen-specific IgG4 in sera ${ }^{(17-20)}$, and activation of regulatory $T$ (Treg) cells and regulatory $B$ (Breg) cells, have been suggested to be involved in the effectiveness of SCIT and SLIT ${ }^{(21-24)}$. However, there have been few studies on the roles of these factors in ILIT. In the present double-blind, placebo-controlled study, we evaluated the clinical effectiveness of ILIT against Japanese cedar pollinosis. Furthermore, we assessed whether the clinical effectiveness of ILIT, in which pollen extracts were intralymphatically injected 3 times before the pollen season, remained in the subsequent two pollen seasons. The effects of ILIT on antigen-specific $\lg \mathrm{E}$ and $\lg \mathrm{G} 4$ levels in sera, and Treg and Breg cells among peripheral blood mononuclear cells (PBMCs) were also evaluated.

\section{Material and methods}

\section{Study population}

Study subjects (20 in total) were recruited among patients at the Otolaryngology Department of Osaka Medical College, Osaka, Japan. The main inclusion criteria were a history of severe Japanese cedar pollen-induced nasal allergy symptoms including sneezing, nasal congestion and secretion, age of 18-70 years, and a detection of serum IgE specific to Cry j1. The main exclusion criteria were serious immunologic diseases, serious cardiovascular diseases, malignant diseases, and uncontrolled severe asthma. Of the 20 patients screened, 18 (6 male and 12 female patients aged 30-65 years) were randomised to receive either Japanese cedar pollen extract or saline (Table 1). The diagnosis was confirmed by the presence of antigen-specific $\lg \mathrm{E}$ antibody (>0.7 IUA/ml) and/or positive nasal provocation test. None of the patients had asthma.

This study was approved by the ethics committees of Osaka Medical College (Osaka, Japan) and Setsunan University (Osaka, Japan), and written informed consent was received from all

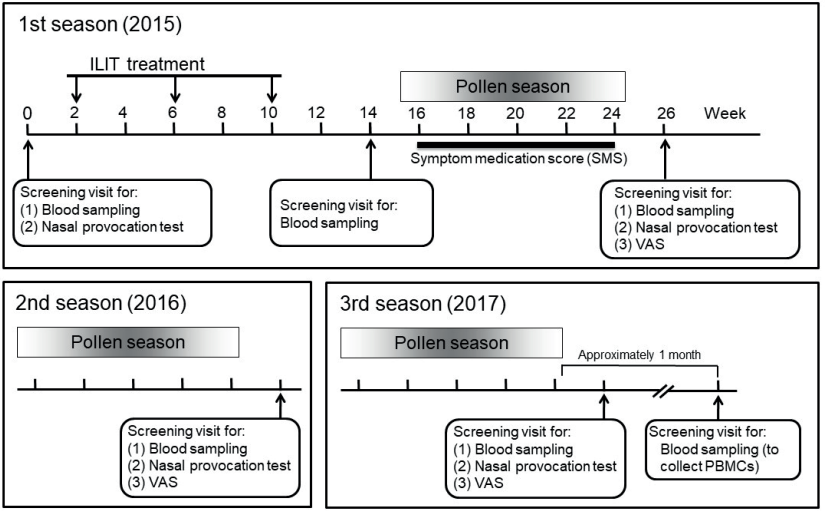

Figure 1. Study design for intralymphatic immunotherapy (ILIT) for patients with Japanese cedar pollinosis, and assessment of the effects in 3 pollen seasons. VAS: visual analogue scale.

participants. The approval numbers are RIN-37 (1568-01) and 2015-032, respectively.

\section{Study objectives and design (Figure 1)}

At the first visit, the nasal provocation test and blood sampling were performed. Approximately 1 week after the first visit, patients in the double-blind study were randomly allocated to receive either placebo $(n=6)$ or active $(n=12)$ intralymphatic treatment. At 2 to 4 visits, the study subjects received three injections with either Japanese cedar pollen extract or saline in a 4-week interval. Blood was again drawn at the first visit and 4 weeks after the last treatment. The symptom medication score (SMS) was noted by the patients during the first pollen season. Approximately 2 weeks after the end of the first pollen season, blood sampling, nasal provocation test, and visual analogue scale (VAS) scoring were conducted. Approximately 2 weeks after the end of the second and third pollen seasons, blood sampling, nasal provocation test, and scoring of VAS were conducted again. Lastly, approximately 1 month after the third pollen season, blood was drawn to detect Treg and Breg cells among PBMCs.

\section{Intralymphatic injections}

A superficial inguinal lymph node was identified to administer $0.1 \mathrm{ml}$ of either Japanese cedar pollen extract (Torii Pharm. Co. Ltd, Tokyo, Japan) at a dose of $20 \mathrm{JAU} /$ patient or saline. Injections were aseptically conducted using a 1-ml hypodermic syringe with a 25 -gauge needle for $1 \mathrm{~min}$. The same side was targeted during all 3 injections. All patients were monitored in the ward for no less than 60 min after each injection. All signs of local and/or systemic reactions in conjunction were recorded by the staff, and patients were then asked to record and report all indications of late reactions during the following $24 \mathrm{~h}$. Intralymphatic injections were controlled and documented by means 
of ultrasonography.

\section{Symptom medication score (SMS)}

During the first season after ILIT (February 3 to March 30, 2015), patients were asked to note their SMS, which was scored by degrees of nasal symptoms, sneezing, rhinorrhoea, and nasal congestion using a 5 -point scale $(0=$ none, $1=$ mild, $2=$ moderate, 3 = severe, and $4=$ very severe) based on the Japanese guidelines for allergic rhinitis ${ }^{(25)}$. The medications were also recorded: the use of antihistamines, mast cell stabilisers, and vasoconstrictors was scored as 1, and topical ocular or nasal steroids as 2 .

\section{Nasal provocation test}

Before ILIT treatment and after the respective pollen seasons, all patients were challenged with Japanese pollen extract. A cotton pellet containing $0.01 \mathrm{ml}$ of $200 \mathrm{JAU}$ of the extract was put on one side of the inferior turbinate mucosa. Five minutes after the challenge, allergic nasal symptoms comprising sneezing or nose itching, aqueous rhinorrhoea, or swelling and pallor of the inferior turbinate mucosa were evaluated (-: none, \pm : 1 symptom, + : 2 symptoms, ++: 3 symptoms, and +++: 3 symptoms and sneezing more than six times). Non-specific hyperresponsiveness was assessed by administration of $0.01 \mathrm{ml}$ diluent to another site of the inferior turbinate mucosa in each patient.

\section{Visual analogue scale (VAS)}

At the end of the respective pollen seasons, patients were asked to score their allergic symptoms during each pollen season in comparison with the symptoms experienced during the season before the start of ILIT. The patients scored their symptoms on a VAS ranging from 0 (unchanged symptoms, no improvement) to 10 (total symptom relief, complete recovery).

\section{Measurement of antibodies}

Venous blood was drawn from all participants at all screening visits. Amounts of allergen-specific lgE, lgG, and lgG4 in the serum were measured using Immulite ${ }^{\circledR}$ total IgE III, Immulite ${ }^{\circledR}$ AlaSTAT IgE II, Immulite ${ }^{\circledast} 2000$ Allergen-Specific IgG, and Immulite ${ }^{\circledast} 2000$ Allergen-Specific IgG4, respectively (Siemens Healthineers, Tokyo, Japan).

\section{FACS analyses for Treg and Breg cells}

Approximately one month after the end of the third pollen season, venous blood was drawn from the patients to collect PBMCs. The methods of detection of Foxp $3^{+}$Treg cells, Tr 1 cells, and Breg cells among PBMCs have been previously reported (22). In brief, PBMCs were isolated from heparinised blood by centrifugation over Histopaque ${ }^{\circledR}-1077$ (Sigma-Aldrich, St. Louis, MO, USA). The isolated PBMCs were incubated with Cry 11 (Cryptomeria japonica 1, 10-5 g/ml, Hayashibara, Okayama, Japan) at $37^{\circ} \mathrm{C}$ for $24 \mathrm{~h}$ in the presence of the protein transport inhibitor

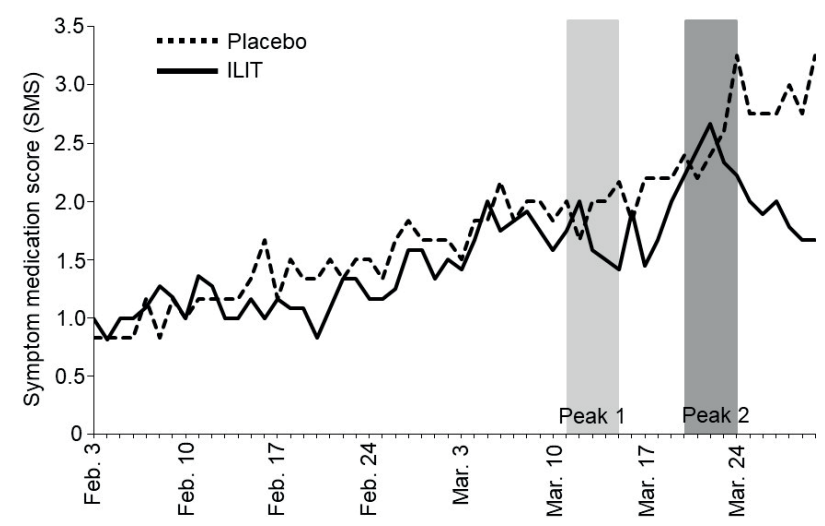

Figure 2. Time-course of the symptom medication score (SMS) in intralymphatic immunotherapy (ILIT)- or placebo-treated patients with Japanese cedar pollinosis. Peak 1 and Peak 2 represent peaks of pollen scattering detected by a system at Osaka Medical College. Each line indicates the mean value of the score of 12 (ILIT-treated) and 6 (placebotreated) patients.

monensin sodium salt ( $2 \mu \mathrm{M}$, Calbiochem, San Diego, CA, USA). For the detection of Foxp $3^{+}$Treg cells, the PBMCs were not treated by the above-mentioned culture conditions.

After washing with $2 \%$ FBS-containing PBS, the cells were treated with Human BD Fc BlockTM (BD Biosciences, San Jose, (A, USA), and cell surface molecules were stained by fluorescein isothiocyanate (FITC)-conjugated anti-human CD3 (HIT3a), PE-conjugated anti-human CD19 (HIB19), peridinin chlorophyll protein (PerCP)-conjugated anti-human CD4 (OKT4), phycoerythrin-cyanin 7 (PE/Cy7)-conjugated anti-human CD45RA (HI100) (all from BioLegend, San Diego, CA, USA), and/or allophycocyanin (APC)-conjugated anti-human CD25 (BC96) (Thermo Fisher Scientific) at $4^{\circ} \mathrm{C}$ for $20 \mathrm{~min}$. After staining the cell surface, the cells were fixed and permeabilised by Fixation/permeabilisation Concentrate (Thermo Fisher Scientific) at room temperature for $1 \mathrm{~h}$. After fixation and permeabilisation, the cells were washed with permeabilisation Buffer (Thermo Fisher Scientific), followed by staining with APC-conjugated anti-human IL-10 (JES3-9D7) (all from BioLegend) and/or PE-conjugated anti-human Foxp3 (236A/E7) (Thermo Fisher Scientific) at room temperature for 1 h. After washing with $2 \%$ FBS-containing PBS, the cells were analysed using FACSAriaTM Fusion (Becton Dickinson, San Jose, (A, USA).

\section{Results}

Adverse events associated with ILIT

Intralymphatic injections with Japanese cedar pollen extracts were conducted 3 times/patient at an interval of 4 weeks for 12 patients (Figure 1). Among 36 total injections, only 5 incidents of mild nasal symptoms were noted in ILIT-treated patients (data not shown). Among placebo-treated patients, nasal symptoms were induced after one of 18 injections. However, other adverse 

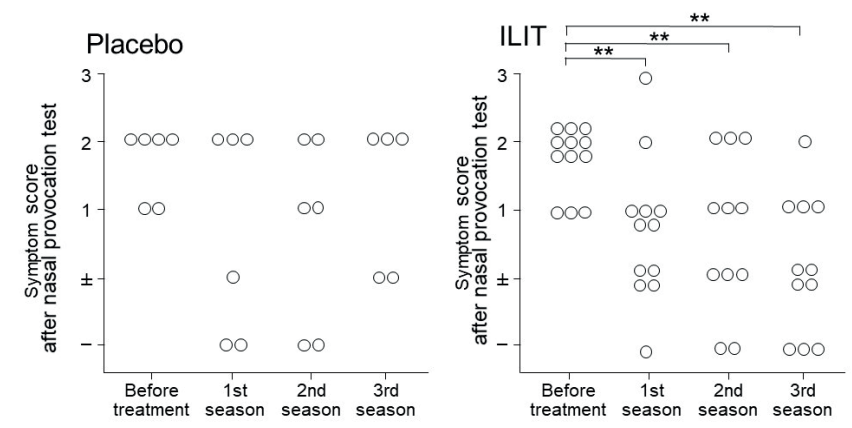

Figure 3. Effects of intralymphatic immunotherapy (ILIT) on the induction of nasal symptoms by nasal provocation test after the respective first, second, and third pollen seasons in patients with Japanese cedar pollinosis. Each point represents the score of respective patients. One placebo-treated patient and one ILIT-treated patient were unable to participate in the assessment after the second and third seasons.

events, including pulmonary symptoms, urticaria and angioedema, and abdominal symptoms, were not observed.

Effects of ILIT on SMS during the first pollen season SMS was recorded during the first pollen season after ILIT. In 2015, a pollen scattering detection system at Osaka Medical College detected two peaks of pollen scattering around Takatsuki city, Osaka, Japan, during May 13-15 and May 23-25 (Figure 2). No statistically significant improvement of SMS was observed, whereas only after the second peak of pollen scattering, SMS tended to be reduced ( $p=0.551$ ) (Figure 2 ).

\section{Longitudinal effects of ILIT on the nasal provocation test and VAS scores}

Nasal provocation tests were performed before and after the first pollen season, and after the second and third pollen seasons. Compared with the nasal provocation test score before the first pollen season, the score of the ILIT-treated patients after the first season was significantly lower (Figure 3). The scores in the second and third seasons were also lower than that before the first season (Figure 3). In contrast, no significant change was noted in the placebo-treated patients during the 3 seasons (Figure 3).

Regarding the VAS scores recorded by placebo- and ILIT-treated patients after the first, second, and third seasons. In the first and second seasons, the VAS score of ILIT-treated patients was significantly higher than that of placebo-treated patients (Figure 4). However, there was no significant difference between the two groups in the third season (Figure 4).

\section{Effects of ILIT on allergen-specific antibody production}

Allergen-specific IgE production slightly increased 4 weeks after the last ILIT treatment in week 14, and then significantly increased in week 23 when the cedar pollen season ended (data not
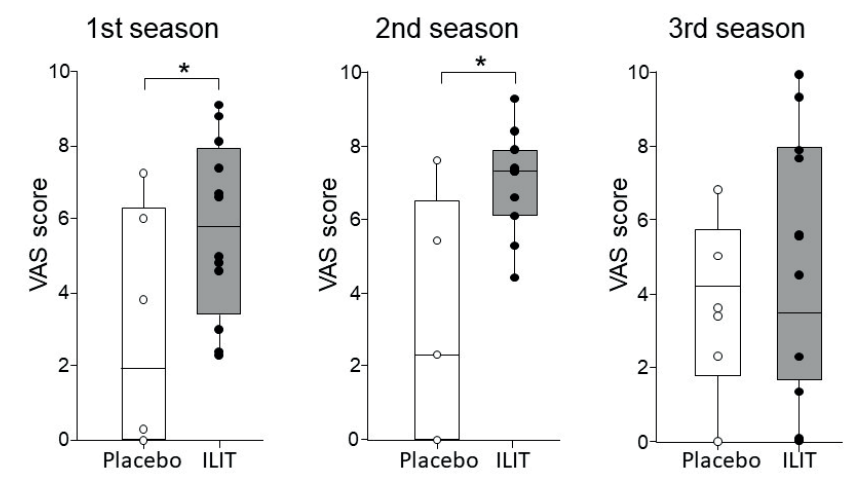

Figure 4. Visual analogue scale (VAS) of patients treated by intralymphatic immunotherapy (ILIT) or placebo after the respective first, second, and third pollen seasons. Each point represents scores of respective patients. The patients were asked to rate the change in their allergic symptoms on a scale ranging from 0 (no improvement) to 10 (complete recovery). One placebo-treated patient and one ILIT-treated patient were unable to participate in the assessment after the second and third seasons.

shown). Antigen-specific lgG production also increased after the ILIT treatment in week 14, followed by a significant decrease in week 23 (data not shown). Regarding placebo-treated patients, the antibody levels did not change (data not shown). On the other hand, allergen-specific lgG4 was not detected (data not shown).

Effects of ILIT on the numbers of Foxp $3^{+}$Treg cells, Tr1 cells, and Breg cells

We next assessed whether the numbers of Foxp $3^{+}$Treg cells, Tr1 cells, and Breg cells were higher in ILIT-treated patients than in placebo-treated Japanese cedar pollinosis patients.

Foxp $3^{+}$Treg cells were analysed as $\mathrm{CD} 25^{+} \mathrm{Foxp}^{+} \mathrm{CD}^{+} \mathrm{T}$ cells (Foxp $3^{+}$Treg cells). There was no significant difference in the number of Foxp $3^{+}$Treg cells among PBMCs between the two groups (data not shown). Tr1 cells among PBMCs were considered to be Foxp3- CD4 $^{+} \mathrm{T}$ cells that produced IL-10 in response to Cry j 1. The number of Tr1 cells in ILIT-treated patients was lower than that in placebo-treated patients, but the difference was not significant (data not shown).

Breg cells among PBMCs were detected as $\mathrm{CD} 19^{+}$cells that produced IL-10 in response to Cry j 1. There was no difference in the number of Breg cells between ILIT- and placebo-treated patients (data not shown).

\section{Discussion}

This is the first, randomised, placebo-controlled, double-blind study of the efficacy and safety of ILIT using a standardised Japanese cedar pollen extract for Japanese cedar pollinosis in Japan. Although this is a preliminary study based on a limited number of patients, ILIT with Japanese cedar pollen extracts demonstrated long-lasting reducing effects on nasal allergic symptoms 
without causing severe adverse events.

SCIT and SLIT are currently performed in immunotherapy for allergic rhinitis. The disadvantages of SCIT include long-term commitment by the patients and the risk of systemic adverse events in relation to allergen administration ${ }^{(3-5)}$. SLIT has been more recently developed and is more patient-friendly, but the frequency and duration of the treatment remain long ${ }^{(26)}$. In this study, we demonstrated that ILIT improved the safety, efficacy, and compliance of SCIT, and reduced the number of injections and cumulative allergen dose.

In three previous reports, ILIT demonstrated clinical improvement in allergic patients against grass or birch pollen and cat dander, although those clinical studies were not double-blind, placebo-controlled ${ }^{(13,15,16)}$. The overall results in our present double-blind, placebo-controlled study in cedar pollinosis patients were consistent with those three studies. In contrast, a double-blind, placebo-controlled study by Witten et al. (27) showed that ILIT exerted no clinical improvement in subjects with grass pollen-induced rhinoconjunctivitis. Reasons for the difference in clinical effectiveness between the former four studies including ours ${ }^{(13,15,16)}$ and the study by Witten et al. ${ }^{(27)}$ are unclear. In the study by Witten et al., the interval of intralymphatic injections was 2 weeks, whereas those in other trials were 4 weeks. This may be a reason for the lack of clinical effects in the study by Witten et al. ${ }^{(27)}$. The administered antigen is presumed to be caught by the antigen-presenting cells, which are subsequently incorporated into the regional lymph nodes, leading to immunological unresponsiveness ${ }^{(14,28)}$. However, in SCIT and SLIT, the amount of antigen incorporated into the regional lymph node should be much lower than that administered subcutaneously or sublingually. Direct antigen administration into the lymph nodes can efficiently induce immunotolerance with a small dose of the antigen. In ILIT for Japanese cedar pollinosis performed in this study, the antigen was administered only 3 times. In contrast, in SCIT for Japanese cedar pollinosis, approximately 54 subcutaneous injections are required over 3 years; therefore, the antigen amount for ILIT was approximately 100 -fold less than that for SCIT.

As inguinal lymph nodes are located just below the skin, they are easy to access and puncture on ultrasonography. In addition, many lymph nodes exist far from large blood vessels. In this study, intralymphatic antigen administration took approximately $1 \mathrm{~min}$ per patient. The cervical lymph node may also be considered as a regional lymph node for nasal tissues, but direct administration has risks of haemorrhage and strong local reactions. We plan to use the cervical lymph node to efficiently deliver the antigen to the regional lymph node closer to the nasal tissues based on the technical experience and safety demonstrated in the present study.

Senti et al. ${ }^{(14)}$ compared the retention time of a protein administered into a lymph node with that administered into subcutane- ous tissue. Isotope-labelled IgG antibody directly administered into a lymph node stayed in the targeted node and surrounding lymph nodes for $24 \mathrm{~h}$. In contrast, when subcutaneously administered, it rapidly dispersed, and only a small amount was incorporated into the regional lymph node. In addition, when an antigen was directly administered into a lymph node in an experimental system using mice, the antigen level in the lymph node was more than 100-times higher than that after subcutaneous administration of the same amount ${ }^{(28)}$. These findings suggested that the administered antigen remains in the lymph node for a prolonged period, and the incorporation into the surrounding lymph nodes is closely associated with the mechanism by which ILIT acts as an efficient immunotherapy.

To develop a new route of administration for immunotherapy, safety is the most important factor. In SCIT, there are risks of adverse reactions, such as anaphylactic reaction, being a disadvantage ${ }^{(9,16,29)}$. However, no severe or systemic adverse reactions were induced by ILIT in any patient in our study. As described above, the total amount of antigen administered in ILIT was much less than that in SCIT, which may be a reason for the absence of adverse reactions. Local reactions at the antigen administration site, such as redness and itching, are inevitable in SCIT. In this study, no swelling of the lymph node to which the antigen was administered was noted. Although bleeding and soreness were not observed at the local injected site, redness of the skin with itching was induced at the injection site in one patient. These adverse effects may be due to local allergic reactions induced by a small volume of leaked antigen around the subcutaneous tissues. The allergic reactions were not induced at the lymph nodes because mast cells are basically absent and there are few blood vessels in the lymph node tissue.

In the study by Senti et al. ${ }^{(13)}$, significantly fewer adverse events developed after ILIT in comparison with SCIT; Grade 1 or 2 mild side reactions were noted during the 4-month period after the initiation of SCIT in 18 patients, and 2 developed asthmatic reactions requiring treatment at a medical institution. On the other hand, in the present study, mild adverse events were induced in only 5 patients treated by ILIT, and no systemic anaphylactic reactions developed. Although simple comparison is not possible because the frequency of antigen administration was different between the present study and the study by Senti et al. ${ }^{(13)}, 3$ intralymphatic injections with the pollen antigen did not induce severe anaphylactic reaction in our pollinosis patients.

When the inhibitory effects were evaluated using SMS in the first pollen season, the score of the ILIT group was lower than that of placebo group. On the VAS-based evaluation, significant therapeutic effects continued for 1 year after the initiation of ILIT. The continued effects over the pollen seasons were observed until the third season when assessed by nasal provocation test. This suggests that ILIT concomitantly exhibits fast-acting therapeutic effects, which last for 1-2 years. Senti et al. ${ }^{(15)}$ investigated 
the amount of antigen necessary to induce nasal symptoms in the nasal provocation test, and found that an approximately 10-times higher amount of antigen than the threshold amount before initiation of ILIT treatment was necessary to induce symptoms in the ILIT treatment group. They performed similar studies 1 and 3 years after the initiation of ILIT or SCIT, and observed a significant increase in the antigen amount required to induce symptoms in both ILIT and SCIT groups, and there was no difference in the degree of increase between the 2 groups. These results reported by Senti et al. ${ }^{(15)}$ suggested that the time required for ILIT to exert its effects is shorter than that of SCIT. These fast-acting effects of ILIT are consistent with our study. The mechanisms underlying the clinical effectiveness of allergen immunotherapy remain unclear. However, some mechanisms, including 1) a decrease in antigen-specific lgE and increase in antigen-specific lgG4, 2) decreases in mast cells, eosinophils, and their mediators in tissues, 3) immune deviation to Th1 cells from Th2 cells, and 4) activation of regulatory $T$ (Treg) cells and regulatory $B$ (Breg) cells, have been suggested ${ }^{(30)}$. Among them, we measured the serum levels of antigen-specific $\lg \mathrm{E}$, IgG, and antigen-specific IgG4, and the numbers of Foxp $3^{+}$Treg cells, $\operatorname{Tr} 1$ cells, and Breg cells in placebo- and ILIT-treated patients.

In patients treated by SCIT for several years, the level of antigenspecific $\lg E$ antibody decreased, and that of $\lg G$, especially $\lg G 4$, was increased in sera ${ }^{(31-33)}$. Regarding changes in antibodies by ILIT in this study, antigen-specific IgE and IgG antibody production increased and decreased, respectively. IgG4 antibody was not detected in sera of either group. Similar to our present findings, in a study on ILIT reported by Hylander et al. ${ }^{(16)}$, IgG4 antibody production was not increased, even though clinical effects were observed. Taken together, the effects of SCIT and ILIT on antibody production differ.

Treg cells and Breg cells, which suppress inflammation, have been considered to play roles in the effects of SCIT, but the details, including the Treg subset, remain unknown. In previous studies, we analysed the numbers of each Treg cell subset and Breg cells among PBMCs collected from pollinosis patients treated by SCIT ${ }^{(24)}$. As a result, the number of Foxp $3^{+} \mathrm{CD} 25^{+}$Treg was not affected by SCIT, and the numbers of Tr1 cells and Breg cells were significantly higher in the SCIT-treated patients than in control patients. On the other hand, in the present study, ILIT did not affect the number of Treg or Breg cells. This is in contrast to the reports by Senti et al. ${ }^{(15)}$ and Martinez-Gomez et al. ${ }^{(28)}$ that ILIT increased IL-10 production, and IL-10-producing T cells and $B$ cells. The reasons for the difference between our present result and other reports ${ }^{(15,28)}$ are unclear. Longitudinal evaluation of Treg and Breg cells may be required over several seasons. Although clinical effectiveness was observed, neither lgG4 production nor Treg/Breg cells were increased in the peripheral blood. A limitation of this study is that we could not have assessed the efficacy of ILIT in a large number of patients, thus we could not raise possible mechanisms of the efficacy at present. Larger clinical studies in the future with cedar pollinosis patients before/after ILIT will determine whether ILIT will be applicable as a potential treatment in individual patients. In addition, murine studies to analyse the mechanisms of ILIT are needed.

\section{Conclusion}

When a small amount of Japanese cedar pollen extract was injected 3 times as ILIT into the inguinal lymph node of patients with Japanese cedar pollinosis, nasal allergic symptoms were effectively reduced without severe adverse effects, and the effects continued for 1-2 years thereafter. Thus, ILIT can be expected as a new allergen-specific immunotherapy for pollinosis. Although the expected value of ILIT seems to be high, further studies are required to assess clinical efficacy, patient acceptability and cost effectiveness in comparison with SCIT and SLIT.

\section{Acknowledgements}

This study was supported by research funding from JSPS KAKENHI Grant Number 17K11370 (to T.T. and T.N.) and Setsunan University (to M.M. and T.N.).

\section{Authorship contribution}

TT and RK were involved in the study design, data collection, data interpretation, data analysis, and writing of the manuscript. SO, YK, MS, Yl, and TI were involved in data collection, data interpretation, and data analysis. MM and TaN were involved in data collection, data interpretation, data analysis, and writing of the manuscript. TT has full access to the data, and is responsible for its integrity and final decision to submit this manuscript. All authors have approved the final version of this manuscript for submission.

\section{Conflict of interest}

The authors declare that no competing interests exist.

\section{References}

1. Kaneko Y, Motohashi Y, Nakamura H, Endo T, Eboshida A. Increasing prevalence of Japanese cedar pollinosis: a meta-regression analysis. Int Arch Allergy Immunol 2005; 136: 365-371

2. Stuck BA, Czajkowski J, Hagner A-E, et al. Changes in daytime sleepiness, quality of life, and objective sleep patterns in seasonal allergic rhinitis: a controlled clinical trial. Allergy Clin Immunol 2004; 113: 663-668.

3. Cox L, Nelson H, Lockey R, et al. Allergen immunotherapy: a practice parameter third update. J Allergy Clin Immunol 2011; 127: S1-55.

4. Settipane RA. Complications of allergic rhi- nitis. Allergy asthma Proc 1999; 20: 209-213. 5. Thompson AK, Juniper E, Meltzer EO. Quality of life in patients with allergic rhinitis. Ann Allergy Asthma Immunol 2000; 85: 338.

6. Schoenwetter WF, Dupclay LJ, Appajosyula S, Botteman MF, Pashos CL. Economic impact and quality-of-life burden of aller- 
gic rhinitis. Curr Med Res Opin 2004; 20 305-317.

7. Gotoh M, Yonekura S, Imai T, et al. Longterm efficacy and dose-finding trial of Japanese cedar pollen sublingual immunotherapy tablet. J Allergy Clin Immunol Pract 2019; 7: 1287-1297.e8.

8. Yonekura S, Okamoto Y, Sakurai D, et al. An analysis of factors related to the effect of sublingual immunotherapy on Japanese cedar pollen induced allergic rhinitis. Allergol Int 2018; 67: 201-208.

9. Okamoto Y, Okubo K, Yonekura S, et al. Efficacy and safety of sublingual immunotherapy for two seasons in patients with Japanese cedar pollinosis. Int Arch Allergy Immunol 2015; 166: 177-188.

10. Frew AJ. Allergen immunotherapy. J Allergy Clin Immunol 2010;125:S306-13.

11. Holgate ST, Polosa R. Treatment strategies for allergy and asthma. Nat Rev Immunol 2008;8:218-30.

12. Hylander $T$, Larsson $O$, Petersson-Westin $U$, et al. Intralymphatic immunotherapy of pollen-induced rhinoconjunctivitis: a double-blind placebo-controlled trial. Respir Res 2016; 17: 10.

13. Senti G, Prinz Vavricka BM, Erdmann I, et al. Intralymphatic allergen administration renders specific immunotherapy faster and safer: a randomized controlled trial. Proc Natl Acad Sci U S A 2008; 105: 17908-17912.

14. Senti G, Johansen P, Kundig TM Intralymphatic immunotherapy. Curr Opin Allergy Clin Immunol 2009; 9: 537-543.

15. Senti G, Crameri R, Kuster D, et al. Intralymphatic immunotherapy for cat allergy induces tolerance after only 3 injections. J Allergy Clin Immunol 2012; 129: 1290-1296.

16. Hylander $T$, Latif $L$, Petersson-Westin $U$, Cardell LO. Intralymphatic allergen-specific immunotherapy: an effective and safe alternative treatment route for pollen-induced allergic rhinitis. J Allergy Clin Immunol 2013; 131: 412-420

17. Gleich GJ, Zimmermann EM, Henderson LL, Yunginger JW. Effect of immunotherapy on immunoglobulin E and immunoglobulin $G$ antibodies to ragweed antigens: A six-year prospective study. J Allergy Clin Immunol 1982; 70: 261-271.

18. Scadding GW, Calderon MA, Shamji MH, et al. Effect of 2 years of treatment with sublingual grass pollen immunotherapy on nasal response to allergen challenge at 3 years among patients with moderate to severe seasonal allergic rhinitis: The GRASS randomized clinical trial. JAMA 2017; 317: 615-625.

19. James LK, Shamji MH, Walker SM, et al. Long-term tolerance after allergen immunotherapy is accompanied by selective persistence of blocking antibodies. J Allergy Clin Immunol 2011; 127: 509-516.e5.

20. Renand A, Archila LD, McGinty J, et al. Chronic cat allergen exposure induces a $\mathrm{TH} 2$ cell-dependent IgG4 response related to low sensitization. J Allergy Clin Immunol 2015; 136: 1627-1635.e13.

21. Suarez-Fueyo A, Ramos T, Galan A, et al. Grass tablet sublingual immunotherapy downregulates the $\mathrm{TH} 2$ cytokine response followed by regulatory T-cell generation. J Allergy Clin Immunol 2014; 133: 130-132.

22. Van De Veen W, Stanic B, Yaman G, et al. $\operatorname{lgG} 4$ production is confined to human IL-10-producing regulatory B cells that suppress antigen-specific immune responses. J Allergy Clin Immunol 2013; 131: 1204-1212.

23. Yamanaka K, Yuta A, Kakeda M, et al. SLIT improves cedar pollinosis by restoring $\mathrm{IL}-10$ production from $\operatorname{Tr} 1$ and Monocytes IL-10 productivity is critical for becoming allergic. Allergol Int 2011; 60: 45-51.

24. Matsuda M, Terada T, Tsujimoto N, et al. Regulatory $T$ and $B$ cells in peripheral blood of subcutaneous immunotherapytreated Japanese cedar pollinosis patients. Immunotherapy 2019; 11: 473-482.

25. Okubo K, Kurono Y, Ichimura K, et al. Japanese guidelines for allergic rhinitis 2017. Allergol Int 2017; 66: 205-219.

26. Canonica GW, Bousquet J, Casale T, et al. Sub-lingual immunotherapy: World Allergy Organization Position Paper 2009. Allergy
2009; 64 Suppl 9: 1-59.

27. Witten $M$, Malling $H J$, Blom $L$, et al. Is intralymphatic immunotherapy ready for clinical use in patients with grass pollen allergy? J Allergy Clin Immunol. 2013 Nov;132(5):1248-1252.

28. Martinez-Gomez JM, Johansen $P$, Erdmann I, Senti G, Crameri R, Kundig TM. Intralymphatic injections as a new administration route for allergen-specific immunotherapy. Int Arch Allergy Immunol 2009; 150: 59-65.

29. Frew AJ. 25. Immunotherapy of allergic disease. J Allergy Clin Immunol 2003; 111: S712-9.

30. Jutel M, Agache I, Bonini S, et al. International Consensus on Allergen Immunotherapy II: Mechanisms, standardization, and pharmacoeconomics. J Allergy Clin Immunol 2016; 137: 358-368.

31. Kay AB. Allergy and allergic diseases. Second of two parts. N Engl J Med 2001; 344: 109-113.

32. Shamji MH, Durham SR. Mechanisms of immunotherapy to aeroallergens. Clin Exp Allergy 2011; 41: 1235-1246.

33. Eifan AO, Shamji MH, Durham SR. Longterm clinical and immunological effects of allergen immunotherapy. Curr Opin Allergy Clin Immunol 2011; 11: 586-593.

Tetsuya Terada, MD, PhD

Department of Otorhinolaryngology

Head and Neck Surgery

Osaka Medical College

2-7 Daigaku-machi

Takatsuki 569-8686

Japan

Tel: +81-726-83-1221

Fax: +81-726-84-6539

E-mail:oto056@osaka-med.ac.jp 